\title{
2019 Reviewers
}

Ádám, Norbert

Ales, Prochazka

Alontseva, Darya

Andoga, Rudolf

Ászity, Sándor

Bakucz, Peter

Balogh, Zoltán

Bán, Krisztián

Bánáti, Anna

Bányai, Tamás

Baranyi, Péter

Bareith, Attila

Bednar, Peter

Bobar, Zoran

Borri, Alessandro

Bozóki, Sándor

Bundzel, Marek

Chinzei, Kiyoyuki

Chiu, Chris

Chopra, Vikram

Ciubotariu, Relu

Čonka, Zsolt

Czakó, Bence Géza

Csanadi, Bertalan

Csapo, Adam

Csato, Laszlo

Csiszár, Csaba

Csiszárik-Kocsir, Ágnes

Csizmadia, Tamas

Dobrzanska, Magdalena

Dombi, József

Domokos, Jozsef

Dömötör, Ferenc

Dragan, Antic

Draganova, Katarina

Duma, Florin
Dvorský, Marián

Eigner, György

Elek, Renata

Fehér-Polgár, Pál

Ferenci, Tamas

Fiorini, Paolo

Fischer, Gregory

Főző, Ladislav

Fullér, Róbert

Galambos, Péter

Gašpar, Vladimír

Genci, Jan

Hanakova, Lenka

Hermann, Gyula

Holicza, Péter

Horvath, Ildiko

Horváth, László

Horváth, Richárd

Hošovský, Alexander

Ionescu, Clara Mihaela

Jaksa, Laszlo

Juan Carlos Garcia Ojeda

Jurado, Francisco

Károly, Artúr István

Kárpáti-Daróczi, Judit

Kasanicky, Tomas

Kátai, László

Katona, Ferenc

Katona, Jozsef

Kazanzides, Peter

Kiss, Gábor

Kluwak, Konrad

Kovacs, George

Kovacs, Szilveszter

Kozma, Tímea

Kronreif, Gernot 
Kuti, József

Kuzsela, László

Lang, Ján

Laššák, Miroslav

Lazanyi, Kornelia

Lewis, Andrew

Li, Yangming

Lipovský, Pavol

Lukács, Pál

Maček, Nemanja

Machova, Renata

Mariani, Andrea

Markus, Zsolt Laszlo

Marovac, Ulfeta

Matiasko, Karol

Menyhart, Jozsef

Mester, Gyula

Mihajlovic, Ivan

Mikula, Martin

Milosavljevic, Cedomir

Moccia, Rocco

Molnar, Ildiko

Molnar, Viktor

Moreira, Pedro

Moya-Albor, Ernesto

Nagy, Dénes Ákos

Nagy, István

Nagy, Tamas

Nedelko, Zlatko

Nedeloni, Marian-Dumitru

Nemes, Csaba

Oancea, Gheorghe

Odry, Peter

Oravec, Jakub

Palumbo, Pasquale

Paniti, Imre

Pawliczek, Adam

Pedro, Rodriguez

Peter, Tamas
Piller, Imre

Piricz, Noémi

Pokorádi, László Károly

Precup, Radu-Emil

Prochazka, Ales

Reicher, Regina Zsuzsánna

Ren, Jin

Rétfalvi, Attila

Rövid, András

Saracevic, Muzafer

Schrötter, Martin

Selvaggio, Mario

Skapinyecz, Róbert

Skokan, Marek

Stanescu, Daniela Natalia

Stević, Željko

Strielkowski, Wadim

Szabo, Ferenc János

Szabolcsi, Robert

Szikora, Péter

Szilagyi, Laszlo

Sztahó, Dávid

Takács, Árpád

Takacs, Istvan

Takacs, Marta

Taneski, Viktor

Tar, József

Tick, Andrea

Tóth-Laufer, Edit

Vámossy, Zoltán

Várlaki, Péter

Vascak, Jan

Velencei, Jolan

Veress, Árpád

Visioli, Antonio

Vlahovic, Nikola

Weber, Gyorgy

Zsoldos, Ibolya 